eligible for blood donation; 58 were ineligible due to age or co-morbidity and 32 were in the induction phase and therefore not eligible.

Prior to the introduction of this service there were 9 regular blood donors amongst this eligible cohort.

$56(85 \%)$ of the potentially eligible patients expressed interest in blood donation and 54 (81\%) applied. The blood service excluded 6 patients for medical reasons and 8 have not attended. This new pathway has therefore resulted in 31 new, and 40 in total, regular blood donors. This cohort has been responsible for the donation of 334 units of blood, 236 of these coming from new donors.

Conclusions 5 year follow-up of this blood donation facilitation service has confirmed that patients with haemochromatosis are willing to be involved in regular blood donation. The number of units donated from these patients has significantly contributed to the regions supply of red cells and could help alleviate ongoing national shortages. The current pathway in Newcastle is now recognised as the 'gold standard' in blood donations amongst patients with haemochromatosis. National rollout of this pathway may have significant impact on the availability of this highly valuable commodity.

\section{ADTU-10 THE SETTING UP AND RUNNING OF A GASTROENTEROLOGY VIRTUAL OUTPATIENT CLINIC FOR NEW REFERRALS}

Kruti Patel ${ }^{*}$, Hakan Kabatas, Emily Savundra, Edward Bosonnet, Bahman Nedjat-Shokouhi. Medefer, London, UK

\subsection{6/gutjnl-2018-BSGAbstracts.451}

Introduction The outpatient services are under increasing pressure across the NHS. A novel approach to virtual outpatients was trialled in this study. The aim was to provide virtual triage and virtual co-ordination of investigations and care of gastroenterology GP referrals, avoiding a physical outpatient visit when deemed clinically safe and appropriate.

Methods All gastroenterology referral, excluding urgent or two-week wait referrals, from Barking, Redbridge and Havering CCG's were included. To support local consultant capacity, we adopted a unique approach by recruiting NHS gastroenterology consultants from around the country to boost local capacity virtually. They were able to review gastroenterology referrals via the bespoke IT platform and work in their own time to meet the demand of the area.

All referrals were initially triaged by a gastroenterology consultant. The consultant could triage the patient to: GP advice only (discharge), outpatient clinic if patients were deemed too complex, and Virtual Hospital pathway. The Virtual Hospital pathway involved a combination of investigations or telephone consultations by junior doctors. All the results were reviewed by the consultant. The outcomes included discharge to GP, further investigations, or outpatient clinic review, for example, for chronic disease management.

Results A total of 1189 of patients were referred to the service from March 2017-January 2018. Of these, 21.19\% were discharged to GP with simple advice, $14.97 \%$ were deemed to complex and reviewed in outpatient clinics, and $63.84 \%$ entered the virtual hospital pathway. The average time taken for the initial consultant triage was 5 hours and 2 min compared to several weeks for an initial outpatient appointment. $99 \%$ of referrals were triaged within 48 hours. $2.5 \%$ of routine referrals were upgraded to urgent and $1 \%$ were upgraded to cancer pathway. Of the 189 patients discharged from the Virtual Hospital, the average time for patients to complete the pathway was 10.11 weeks. No clinically adverse incidents have been reported. The service has been well received by patients, and $\sim 1 \%$ of patients requested an outpatient visit.

Conclusions The virtual hospital has created a safe and efficient healthcare pathway, utilising technology that significantly reduces hospital footfall, reducing patient waiting times and delivers system-based cost savings. We have identified a sustainable solution to improve and meet increasing demand despite limited capacity within the NHS. The Virtual Hospital demonstrates that majority of patients under virtual specialist care can be managed within the community setting or virtually. Early triage also helps reduce clinical risk for patients who would have otherwise waited several weeks for a clinic appointment.

\section{PTU-073 A THOUSAND CAPSULES: SIX YEARS' EXPERIENCE FROM A DISTRICT GENERAL HOSPITAL IN ENGLAND}

${ }^{1}$ Hussam ME Ahmed*, ${ }^{1}$ Burhan Uddin, ${ }^{1}$ Kapil Kapur, ${ }^{2}$ Anastasios Koulaouzidis, ${ }^{1}$ Elmuhtady Said. ${ }^{1}$ Barnsley District General Hospital, Barnsley, UK; ${ }^{2}$ Royal Infirmary of Edinburgh, Edinburgh, UK

\subsection{6/gutjnl-2018-BSGAbstracts.452}

Introduction The diagnostic role of capsule endoscopy CE has rapidly evolved over the past two decades and is currently regarded as one of the gold standard test to investigate the small bowel. Although most publications are from tertiary referral centres, there is a paucity of data on feasibility and clinical utility of CE in a district General hospital (DGH) setting in the United Kingdom (UK). We aimed to evaluate the capsule endoscopy service in our Hospital since it has been established in 2011.

Methods We retrospectively reviewed and analysed the CE reports of all patients who had the test between April 2011 and April 2017. Exclusion criteria: Retained capsule, difficulty to swallow capsule and inadequate views due to poor bowel prep. We assessed demographics, indications, outcome, complications and completion of the test among the whole cohort. We also looked at the diagnostic yield (DY).

Results In the aforementioned period, Small bowel CE was performed on 1029 patients. 71 (6.9\%) patients were excluded as per exclusion criteria. 958 (528F/430M; Mean age 59 years, Range: 17-92) patients' reports were reviewed and analysed. OGIB was recorded as the main indication $81.4 \%$ (iron deficiency anaemia, IDA: $74.9 \%(n=718)$, overt bleeding $6.5 \%(n=62))$. Other indications included investigation of small bowel Crohn's disease (CD) 10.3\% ( $n=99)$, weight loss: $1.7 \% \quad(n=16)$, angiodysplasia: $1.3 \% \quad(n=12)$, abdominal pain: 2\% $(n=19)$ and others: $7.2 \%(n=69)$. Complete small bowel examination was achieved in 902 patients (94.2\%). 56 patients (5.8\%) had sub optimal views due to rapid transit time. The test was normal in 61\% $(n=581)$. Small bowel angioectasia was detected in $13.8 \% \quad(n=132)$. Other findings included apthoid ulcers: $11.8 \% \quad(n=113)$, inflammation and oedema suggestive of Crohn's disease in $8 \%$ $(\mathrm{n}=76)$, polypoidal lesion $1.5 \%(\mathrm{n}=17)$, colonic pathologies $1.5 \%(\mathrm{n}=14)$ and others $5.8 \%(\mathrm{n}=56)$. Patients with suspected small bowel Crohn's disease had initial patency capsule to prevent lodgement. Retention was recorded in $3 \%$ of patients $(n=3)$ due to strictures. One patient's stricture improved with 